\title{
[Technical Note] \\ Purely-Algebraic versus VEKM Methods for Solving Big Boolean Equations
}

\author{
Ali Muhammad Ali Rushdi and Motaz Hussain Amashah \\ King Abdulaziz University, ECE, \\ P. O. Box 80204, Jeddah 21589, Saudi Arabia \\ arushdi@kau.edu.sa
}

\begin{abstract}
Manual methods of solving big Boolean equations include purely-algebraic methods and methods utilizing the variable-entered Karnaugh map (VEKM). The VEKM techniques are demonstrated to be at least competitive with (and occasionally superior to) purelyalgebraic techniques, since the VEKM techniques (a) include purelyalgebraic methods as special cases, and (b) have a better control on the minimality of the pertinent function representations, and hence are more capable of producing more compact general solutions.
\end{abstract}

Keywords: Solution of big Boolean equations, VEKM techniques, Purely-algebraic techniques.

\section{Introduction}

There are two prominent classes of methods used in the manual solution of big Boolean equations, namely, the class of purely- algebraic methods and the class of methods utilizing the variable-entered Karnaugh map (VEKM). This note compares the competitiveness of these two classes of methods, and sets the record straight by showing that the downgrading of VEKM methods in ${ }^{[1]}$ is unjustified and inaccurate. The importance of this note is that it offers decisive facts that oppose opinions set forth by Professor Sergiu Rudeanu, who is widely and undisputedly recognized in pertinent circles as the father of modern Boolean equations. 
Rudeanu states in ${ }^{[2]}$ and reiterates in Ref. $[1,3]$ that 'the algebraic theory of Boolean equations is very strong and provides various efficient methods for solving them.' For him, the '[map] procedures are, in fact, nothing but practical ways of organizing the [algebraic] methods,' [2], and 'the map methods do not take advantage of the results provided by the algebraic theory, and besides, are limited to the case of the twoelement ...or a finite Boolean algebra.' To support his point, he resumed in Ref. [1] an equation solved in Ref. [4, 5] by what he calls a tabular method, solved it algebraically, and recovered the compact solution in Ref. [4, 5].

We have several remarks on the comparisons between algebraic and tabular methods stated in Ref. [1-3]. First, we have to declare and stress our due respect to algebraic methods. They constitute the pillars upon which other methods are built and they are inescapable in every implementation detail. We agree that map procedures add nothing to algebraic methods beyond being a practical way of organizing them. However, such practicality should be not belittled, as it adds pictorial insight and divide-and-conquer strategies that facilitate the manipulations, make them faster and less error prone, and probably produce more compact results. We also disagree on the lumping of map methods under the general category of tabular methods. From a nowdominant engineering point of view, map (specifically, Karnaugh map) methods are distinguished from tabular methods ${ }^{[6]}$. Though the Karnaugh map is essentially a truth table, it has typically a twodimensional rather than a one-dimensional layout. More importantly, the ordering of rows and columns in a Karnaugh map is a reflected grey (instead of binary) ordering; thereby endowing the map cells by useful adjacency properties ${ }^{[4-7]}$.

In addition, the map referred to in Ref. [1] is not a classical Karnaugh map (CKM), but a variable-entered one $\left.{ }^{[5,7-14}\right]$. The variableentered Karnaugh map (VEKM) does not offer a purely map method, as it is semi algebraic in nature ${ }^{[14]}$. The VEKM is the natural map for representing finite big Boolean functions that are not necessarily twovalued functions ${ }^{[4,8]}$. A Boolean function of $n$ variables has $2^{n}$ VEKM representations (depending on the choice of map and entered variables) ranging from a CKM (of $\mathrm{n}$ map variables and 0 entered variables), and a purely-algebraic expression (of 0 map variables and $n$ entered variables). 
This means clearly that a purely-algebraic expression is a special case of a VEKM representation.

To summarize our remarks, we admit that the VEKM cannot handle infinite Boolean algebras, but it serves as a natural map for finite big Boolean algebras. There is no point of claiming that algebraic methods are superior to VEKM methods, since the latter include the former as special cases. On the contrary, VEKM methods, being semi algebraic in nature, can always take full advantage of the results provided by the algebraic theory. They have a better control on the minimality of the pertinent function representation. Specifically, we study the algebraic method of Rudeanu in Ref. [1] and show that it derives its simplicity from sacrificing a degree of freedom offered by an earlier doubleinequality solution due to Rudeanu ${ }^{[2]}$; thereby using a particular completely-specified instant of that solution. Hence, it secures local minimality over a set of chosen coefficients and not over the more basic set of pertinent variables and generators. Therefore, this method is not always as efficient as a general VEKM method, which seeks global minimality based on an incompletely-specified adaptation of the doubleinequality solution of Rudeanu ${ }^{[2]}$. This means that VEKM solutions are at least as good as purely-algebraic ones (as happened in Ref. [1]), but are occasionally better (as will be demonstrated herein).

The rest of this note is organized as follows. Section 2 gives a brief outline of the double interval-solution of Boolean equations ${ }^{[2-5,10]}$, the use of its incompletely-specified version for the VEKM solution in Ref. $[5,10]$, and the purely-algebraic approach in Ref. [1]. Section 3 assesses the purely-algebraic approach ${ }^{[1]}$ as a special case of the VEKM solution in Ref. [5]. Section 4 presents the two solutions for a Boolean equation of one variable in a big Boolean algebra. The VEKM solution is much simpler than the purely-algebraic one, and hence serves as a more efficient generator of all particular solutions. Section 5 concludes this note.

\section{Subsumptive General Solutions}

In this section, we review the classical technique of constructing subsumptive general solutions for a Boolean system of equations. More details can be found in Ref. $[4,5,10]$, and a formal proof is available in Ref. [2]. 
An n-variable Boolean system on a Boolean algebra $\mathbf{B}$ is a set of $k$ simultaneously asserted equations. This system is equivalent to the single equation

$$
f(\mathbf{X})=0
$$

where $\mathbf{X}=\left[X_{1}, X_{2}, \ldots, X_{n}\right]^{\mathrm{T}}$ is a vector of $n$ components $X_{\mathrm{i}}$ each belonging to the Boolean carrier $\mathbf{B}$. The subsumptive solution is obtained by constructing the eliminants

$f_{n}\left(X_{1}, X_{2}, \ldots, X_{n}\right), \ldots, f_{i}\left(X_{1}, X_{2}, \ldots, X_{i-1}, X_{i}\right), \ldots, f_{2}\left(X_{1}, X_{2}\right), f_{1}\left(X_{1}\right), f_{0}$

by setting $f_{n}=f$ and using the recursion

$$
f_{i}\left(X_{1}, X_{2}, \ldots, X_{i-1}\right)=\left(f_{i} / \bar{X}_{i}\right) \wedge\left(f_{i} / X_{i}\right)=0, \quad i=n, n-1, \ldots, 1
$$

Note that $f_{i-1}$ is the conjunctive eliminant of $f_{i}$ with respect to the singleton $\left\{X_{i}\right\}^{[4]}$. This means that $f_{i-1}$ is a conjunction of the two ratios or subfunctions

$$
\begin{aligned}
& f_{i} / \bar{X}_{i}=f_{i}\left(X_{1}, X_{2}, \ldots, X_{i-1}, 0\right) \\
& f_{i} / X_{i}=f_{i}\left(X_{1}, X_{2}, \ldots, X_{i-1}, 1\right)
\end{aligned}
$$

obtained from $f_{i}$ by setting or restricting $\mathrm{X}_{\mathrm{i}}$ in it to 0 and to 1 , respectively. For simplicity, these two ratios will be denoted by $f_{i}(0)$ and $f_{i}(1)$, respectively. The recursive implementation of (2) results in a successive elimination of variables, and a transformation of the problem (1) of solving a single equation of $n$ variables to that of solving $n$ equations of one variable each. The solution requires a separate consistency condition

$$
f_{0}=0
$$

plus expressing each of the pertinent variables as an interval of functions of the preceding variables, namely:

$$
s_{i}\left(X_{1}, X_{2}, \ldots, X_{\mathrm{i}-1}\right) \leq \mathrm{X}_{\mathrm{i}} \leq t_{i}\left(X_{1}, X_{2}, \ldots, X_{\mathrm{i}-1}\right) \quad i=1,2, \ldots, n
$$

where the functions $s_{i}$ and $t_{i}$ can be expressed as completely-specified Boolean functions. However, to leave room for further simplifications, they are expressed as incompletely-specified Boolean functions (ISBFs) in the interval or double-inequality form ${ }^{[4]}$

$$
\begin{gathered}
f_{i}(0) \bar{f}_{i}(1) \leq s_{i} \leq f_{i}(0) \\
\bar{f}_{i}(1) \leq t_{i} \leq f_{i}(0) \vee \bar{f}_{i}(1)
\end{gathered}
$$


The form of the general solution above allows all the particular solutions of (1), and nothing else, to be generated as a tree.

The key step in the VEKM subsumptive solution is to convert the double-interval conditions (6) into the incompletely-specified expressions ${ }^{[5,10]}$

$$
\begin{gathered}
s_{i}=f_{i}(0) \bar{f}_{i}(1) \vee d\left(f_{i}(0)\right) \\
t_{i}=\bar{f}_{i}(1) \vee d\left(f_{i}(0)\right)
\end{gathered}
$$

and then utilize the VEKM to implement the ANDing operations in (2), the complementation, ANDing, and ORing operations in (6), and the minimization suggested by $(7)^{[5,10-14]}$.

On the other hand, the purely-algebraic method in Ref. [1] for solving (1) starts by writing the function $f(\mathbf{x})=f_{\mathrm{n}}(\mathbf{x})$ of $\mathrm{n}$ variables as

$$
f_{n}(x)=\mathrm{A}_{\mathrm{n}} \mathrm{X}_{\mathrm{n}} \vee \mathrm{B}_{\mathrm{n}} \overline{\mathrm{X}}_{\mathrm{n}} \vee \mathrm{C}_{\mathrm{n}}=0
$$

where the coefficients $A_{n}=A_{n}\left(X / X_{n}\right), B_{n}=B_{n}\left(X / X_{n}\right), C_{n}=C_{n}\left(X / X_{n}\right)$ are functions of the (n-1) remaining variables $\left(X / X_{n}\right)=\left(X_{1}, X_{2}, \ldots, X_{n-1}\right)$. The subsumptive solution for $X_{n}$ in terms of the other $(n-1)$ variables is provided by the double inequality

$$
\mathrm{B}_{\mathrm{n}} \leq \mathrm{X}_{\mathrm{n}} \leq \overline{\mathrm{A}}_{\mathrm{n}}
$$

provided the following consistency condition is satisfied

$$
f_{\mathrm{n}-1}(\mathrm{x})=\mathrm{A}_{\mathrm{n}} \mathrm{B}_{\mathrm{n}} \vee \mathrm{C}_{\mathrm{n}}=0
$$

Thus the solution of (8) is provided partially by (9) for $\mathrm{X}_{\mathrm{n}}$, and is reduced to the solution of (10) for the remaining (n-1) variables. The iteration of the above procedure leads to a successive elimination of variables and the production of a subsumptive solution for each variable in terms of the earlier variables, in addition to a final consistency condition that involves no variables but involves constants of the underlying Boolean algebra.

\section{Assessment of the Purely-Algebraic Method}

The algebraic method in Ref. [1] produces a sequence of equations $f_{i}=0,(i=n$ down to $i)$ where $f_{i}=f_{i}\left(\mathrm{X}_{1}, \mathrm{X}_{2}, \ldots, \mathrm{X}_{\mathrm{i}-1}, \mathrm{X}_{\mathrm{i}}\right)$ is

$f_{i}=A_{i} X_{i} \vee B_{i} \bar{X}_{i} \vee C_{i}=\left(A_{i} \vee C_{i}\right) X_{i} \vee\left(B_{i} \vee C_{i}\right) \bar{X}_{i}=f_{i}\left(1_{i}\right) X_{i} \vee f_{i}\left(0_{i}\right) \bar{X}_{i}$ 
Equation (11) is a Boole-Shannon expansion of the function $f_{\mathrm{i}}$, in which the subfunctions $f_{\mathrm{i}}\left(1_{\mathrm{i}}\right)$ and $f_{\mathrm{i}}\left(0_{\mathrm{i}}\right)$ are:

$$
\begin{aligned}
& f_{i}\left(1_{\mathrm{i}}\right)=f_{i}\left(\mathrm{X}_{1}, \mathrm{X}_{2}, \ldots, \mathrm{X}_{\mathrm{i}-1}, 1\right)=\mathrm{A}_{\mathrm{i}} \vee \mathrm{C}_{\mathrm{i}} \\
& f_{i}\left(0_{\mathrm{i}}\right)=f_{i}\left(\mathrm{X}_{1}, \mathrm{X}_{2}, \ldots, \mathrm{X}_{\mathrm{i}-1}, 0\right)=\mathrm{B}_{\mathrm{i}} \vee \mathrm{C}_{\mathrm{i}}
\end{aligned}
$$

Now employing the incompletely-specified definitions (7a) and (7b) for $s_{i}$ and $t_{i}$, we obtain

$$
\begin{gathered}
\mathrm{s}_{\mathrm{i}}=\overline{\mathrm{A}}_{\mathrm{i}} \overline{\mathrm{C}}_{\mathrm{i}} \mathrm{B}_{\mathrm{i}} \vee \mathrm{d}\left(\mathrm{B}_{\mathrm{i}} \vee \mathrm{C}_{\mathrm{i}}\right) \\
\mathrm{t}_{\mathrm{i}}=\overline{\mathrm{A}}_{\mathrm{i}} \overline{\mathrm{C}}_{\mathrm{i}} \vee \mathrm{d}\left(\mathrm{B}_{\mathrm{i}} \vee \mathrm{C}_{\mathrm{i}}\right)
\end{gathered}
$$

The expressions for $\mathrm{s}_{\mathrm{i}}$ and $\mathrm{t}_{\mathrm{i}}$ can be simplified to

$$
\begin{gathered}
\mathrm{s}_{\mathrm{i}}=\mathrm{B}_{\mathrm{i}} \\
\mathrm{t}_{\mathrm{i}}=\overline{\mathrm{A}}_{\mathrm{i}} \overline{\mathrm{C}}_{\mathrm{i}} \vee \mathrm{d}\left(\mathrm{B}_{\mathrm{i}} \vee \mathrm{C}_{\mathrm{i}} \vee \overline{\mathrm{A}}_{\mathrm{i}}\right)=\overline{\mathrm{A}}_{\mathrm{i}}
\end{gathered}
$$

and hence, we can replace $(5 \mathrm{~b})$ by:

$$
\mathrm{B}_{\mathrm{i}} \leq \mathrm{X}_{\mathrm{i}} \leq \overline{\mathrm{A}}_{\mathrm{i}}, \quad \mathrm{i}=\mathrm{n},(\mathrm{n}-1), \ldots, 2,1
$$

in agreement with formula (9), obtained earlier through the purelyalgebraic method.

We note that the freedom allowed by the intervals in (6) or the don't-care conditions in (7) is not fully utilized in the purely-algebraic method in general, since it strives to achieve greedy or local minimality over all possible choices of the coefficients $\mathrm{A}_{\mathrm{i}}, \mathrm{B}_{\mathrm{i}}$ and $\mathrm{C}_{\mathrm{i}}$. This kind of local minimality is sub-optimal compared with global minimality over the underlying set of variables and generators, that is suggested by (6) or (7) and fully employed in our subsumptive VEKM solution (Section 2). Though the purely-algebraic method in Ref. [1] is generally sub-optimal, it achieved the minimal solution for the example in Ref. [4, 5]. However, it fails to obtain minimality for many other examples, including the illustrative example of the next section.

\section{An Illustrative Example}

Consider the function $f(\mathrm{x}): \mathbf{B}_{\mathrm{n}} \rightarrow \mathbf{B}_{\mathrm{n}}$, given by

$$
f(X)=a \bar{b} X \vee \bar{a} c X \vee \bar{b} d X \vee \bar{a} b \bar{X} \vee \bar{a} d \bar{X} \vee \bar{b} c \bar{X} \vee a b=0
$$


Here, we assume that $n=2^{m}=65536$, where $m=2^{4}=16$ is the number of atoms of the underlying Boolean carrier $\mathbf{B}_{\mathrm{n}}$. These atoms constitute the elements of the free Boolean algebra $F B(\mathrm{a}, \mathrm{b}, \mathrm{c}, \mathrm{d}){ }^{[4,13,14]}$. Figure 1(a) shows a VEKM representation of $f(\mathrm{X})$ obtained via a BooleShannon expansion of $f(\mathrm{X})$ with respect to $\mathrm{X}$. Figure 1(b) simplifies the VEKM entries of Fig. 1(a) via consensus generation and absorption. Figure 2 illustrates the steps needed in the VEKM solution of $f(\mathrm{x})=0$, which is

$$
\mathrm{b} \leq \mathrm{X} \leq \overline{\mathrm{a}}
$$

together with the consistency condition

$$
c \vee a b \vee \bar{a} \bar{b} d=0
$$

or equivalently:

$$
\mathrm{c}=0, \mathrm{ab}=0, \overline{\mathrm{a}} \overline{\mathrm{b}} \mathrm{d}=0
$$

which means that the underlying Boolean algebra has 11 of its atoms nullified, and hence it collapses to the Boolean algebra $\mathbf{B}_{32}$ of the 5 atoms $\bar{a} \bar{b} \bar{c} \bar{d}, \bar{a} b \bar{c} \bar{d}, \bar{a} b \bar{c} d, a \bar{b} \bar{c} \bar{d}$, and a $\bar{b} \bar{c} d$ (subject to conditions (21b)).

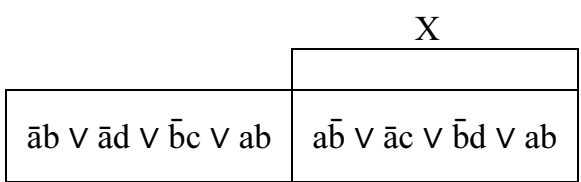

(a) $f(\mathrm{x})$

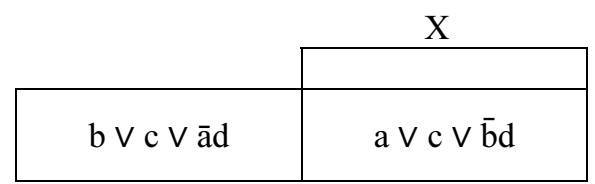

(b) $f(\mathrm{x})$

Fig. 1. VEKM representations of the function $f$ in (19).

\begin{tabular}{|c|}
\hline $\mathrm{b} \vee \mathrm{c} \vee \overline{\mathrm{a} d}$ \\
\hline$f(0)$ \\
\hline $\begin{array}{c}\overline{\mathrm{a}} \mathrm{b} \overline{\mathrm{c}} \vee \\
\mathrm{d}(\mathrm{b} \vee \mathrm{c} \vee \overline{\mathrm{a}} \mathrm{d})\end{array}$ \\
$s_{l}=\mathrm{b}$
\end{tabular}

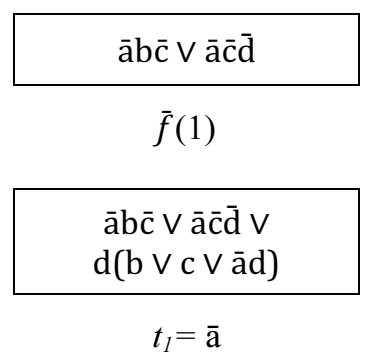

$c \vee a b \vee \bar{a} \bar{b} d$

$f_{0}$

Fig. 2. Steps of the VEKM subsumptive solution of $f(x)=0$. 
On the other hand, the purely-algebraic solution in Ref. [1] proceeds as follows:

$$
\begin{aligned}
& f=f_{1}(\mathrm{x})=(\mathrm{a} \overline{\mathrm{b}} \vee \overline{\mathrm{a}} \mathrm{c} \vee \overline{\mathrm{b}} \mathrm{d}) \mathrm{X} \vee(\overline{\mathrm{a}} \mathrm{b} \vee \overline{\mathrm{a}} \mathrm{d} \vee \overline{\mathrm{b}} \mathrm{c}) \overline{\mathrm{X}} \vee \mathrm{ab}=0 \\
& \mathrm{~A}_{1}=\mathrm{a} \overline{\mathrm{b}} \vee \overline{\mathrm{a}} \mathrm{c} \vee \overline{\mathrm{b}} \mathrm{d}, \mathrm{B}_{1}=\overline{\mathrm{a}} \mathrm{b} \vee \overline{\mathrm{a}} \mathrm{d} \vee \overline{\mathrm{b}} \mathrm{c}, \mathrm{C}_{1}=\mathrm{ab} \\
& \overline{\mathrm{A}}_{1}=(\overline{\mathrm{a}} \vee \mathrm{b})(\mathrm{a} \vee \bar{c})(\mathrm{b} \vee \overline{\mathrm{d}})=(\mathrm{b} \vee \overline{\mathrm{a}} \overline{\mathrm{d}})(\mathrm{a} \vee \bar{c})=\mathrm{ab} \vee \mathrm{b} \bar{c} \vee \overline{\mathrm{a}} \overline{\mathrm{c}} \overline{\mathrm{d}}
\end{aligned}
$$

The algebraic solution is

$$
\mathrm{B}_{1} \leq \mathrm{X} \leq \overline{\mathrm{A}}_{1}
$$

or

$$
(\bar{a} b \vee \bar{a} d \vee \bar{b} c) \leq X \leq(a b \vee b \bar{c} \vee \bar{a} \bar{c} \bar{d})
$$

with the consistency condition

$$
\begin{aligned}
f_{0} & =\mathrm{A}_{1} \mathrm{~B}_{1} \vee \mathrm{C}_{1} \\
& =(\mathrm{a} \overline{\mathrm{b}} \vee \overline{\mathrm{a}} \mathrm{c} \vee \overline{\mathrm{b}} \mathrm{d})(\overline{\mathrm{a}} \mathrm{b} \vee \overline{\mathrm{a}} \mathrm{d} \vee \overline{\mathrm{b}} \mathrm{c}) \vee \mathrm{ab} \\
& =\mathrm{a} \overline{\mathrm{b}} \mathrm{c} \vee \overline{\mathrm{a}} \mathrm{b} \vee \overline{\mathrm{a}} \mathrm{c} \vee \overline{\mathrm{a}} \overline{\mathrm{b}} \mathrm{c} \vee \overline{\mathrm{a}} \overline{\mathrm{b}} \mathrm{d} \vee \overline{\mathrm{b}} \mathrm{dc} \vee \mathrm{ab} \\
& =\mathrm{c} \vee \mathrm{ab} \vee \overline{\mathrm{a}} \overline{\mathrm{b}} \mathrm{d} \\
& =0
\end{aligned}
$$

The subsumptive solution (20) is clearly much simpler than its counterpart in (24), and hence is expected to be more efficient as a generator of particular solutions. The two solutions are of course equivalent subject to the common consistency condition in (21) or (25).

In passing, we note that the parametric-solution method in Ref. [13, 14] predicts that the number of particular solutions of $f(x)=0$ is just two. Since the limits of the interval in (20) are not equal $\{b \neq \bar{a}\}$, or $\{b \bar{a} \vee \bar{b} a \neq$ 0 , they represent these two solutions as $X=b$ and $X=\bar{a}$. In fact,

$$
\begin{aligned}
& f(\mathrm{~b})=\overline{\mathrm{a}} \mathrm{cb} \vee \overline{\mathrm{a}} \mathrm{d} \overline{\mathrm{b}} \vee \overline{\mathrm{b}} \mathrm{c} \vee \mathrm{ab}=\mathrm{c} \vee \mathrm{ab} \vee \overline{\mathrm{a}} \overline{\mathrm{b}} \mathrm{d}=0 \\
& f(\overline{\mathrm{a}})=\overline{\mathrm{a}} \mathrm{c} \vee \overline{\mathrm{a}} \overline{\mathrm{b}} \mathrm{d} \vee \mathrm{a} \overline{\mathrm{b}} \mathrm{c} \vee \mathrm{ab} \quad \mathrm{c} \vee \mathrm{ab} \vee \overline{\mathrm{a}} \overline{\mathrm{b}} \mathrm{d}=0
\end{aligned}
$$

The fact that there are only two particular solutions $X=b$, and $X=$ $\overline{\mathrm{a}}$ is confirmed by the observation that there is no lattice point in the final $\mathrm{B}_{32}$ carrier between points $\mathrm{b}$ and $\overline{\mathrm{a}}$. When lattice point $\mathrm{b}$ is augmented by the atom $\bar{a} \bar{b} \bar{c} \bar{d}$ (subject to condition (21b)), then lattice point $\bar{a}$ is directly reached. 


\section{Conclusions}

In this note, we made a detailed comparison of the two prominent classes of methods used in the manual solution of big Boolean equations, viz., the class of purely- algebraic methods and the class of VEKM methods. Though VEKM methods can be generally categorized as semialgebraic methods or as tabular methods, they have their own distinguishing characteristics to warrant classifying them as a separate class of methods.

We stressed that the VEKM is the natural map for the underlying finite big Boolean algebras. We pointed out that VEKM techniques include purely-algebraic techniques as special cases and supplement them with a practical divide-and-conquer strategy. Hence, we anticipated that VEKM techniques should never be inferior to purely-algebraic techniques, and can frequently produce better results since VEKM techniques naturally and easily secure minimality over the basic set of pertinent variables and generators, while a purely-algebraic technique seek a restricted sort of minimality over a set of chosen coefficients. We supported our argument by an illustrative example in which a VEKM technique was much easier to implement and produced a much more compact solution (a minimal solution).

In conclusion, we note that algebraic methods are useful in the initial study of the subject to maintain the rigor and set the theoretical framework. Map methods (VEKM methods, in particular) complement algebraic methods, as they provide pictorial insights, require easier shortcut manipulations, and produce much more compact general solutions.

\section{References}

[1] Rudeanu, S., Algebraic methods versus map methods of solving Boolean equations, International Journal of Computer Mathematics, 80 (7): 815-817 (2003).

[2] Rudeanu, S., Boolean Functions and Equations, North-Holland Publishing Company \& American Elsevier, Amsterdam, the Netherlands (1974).

[3] Rudeanu, S., Lattice Functions and Equations, Springer, London, UK (2001).

[4] Brown, F.M., Boolean Reasoning: The Logic of Boolean Equations, Kluwer Academic Publishers, Boston, MA, USA (1990).

[5] Rushdi, A.M., Using Variable-Entered Karnaugh Maps to Solve Boolean Equations, International Journal of Computer Mathematics, 78 (1): 23-38 (2001).

[6] Muroga, S., Logic Design and Switching Theory, John Wiley and Sons, New York, NY, USA (1979). 
[7] Rushdi, A.M., Karnaugh map, Encyclopaedia of Mathematics, Supplement Volume I, M. Hazewinkel (editor), Boston, Kluwer Academic Publishers, pp. 327-328 (1997). Available at http://www.encyclopediaofmath.org/index.php/Karnaugh_map.

[8] Rushdi, A.M., Symbolic reliability analysis with the aid of variable-entered Karnaugh maps, IEEE Transactions on Reliability, R-32 (2): 134-139 (1983).

[9] Rushdi, A.M., Performance indexes of a telecommunication network, IEEE Transactions on Reliability, R-37 (1): 57-64 (1988).

[10] Rushdi, A.M., Efficient solution of Boolean equations using variable-entered Karnaugh maps, Journal of King Abdulaziz University: Engineering Sciences, 15 (2): 21-29 (2004).

[11] Rushdi, A.M., Improved variable-entered Karnaugh map procedures, Computers and Electrical Engineering, 13 (1): 41-52 (1987).

[12] Rushdi, A.M. and H. A. Al-Yahya, A Boolean minimization procedure using the variableentered Karnaugh map and the generalized consensus concept, International Journal of Electronics, 87 (7): 769-794 (2000).

[13] Rushdi, A.M. and M. H. Amashah, Parametric general solutions of Boolean equations via variable-entered Karnaugh maps, Journal of Qassim University: Engineering and Computer Sciences, 3 (1): 59-71 (2010).

[14] Rushdi, A.M. and M.H. Amashah, Using variable-entered Karnaugh maps to produce compact parametric general solutions of Boolean equations, International Journal of Computer Mathematics, 88 (15): 3136-3149 (2011). 


\title{
مقارنة الطرائق الجبرية البحتة وطرائق الخريطة متغيرة المحتويات عند حل المعادلات البولانية الكبيرة
}

\author{
علي محمد علي رشدي و معتز حسين عماشة \\ قسم الهنسة الكهريائية وهندسة الحاسبات، جامعة الملك عبد العزيز،

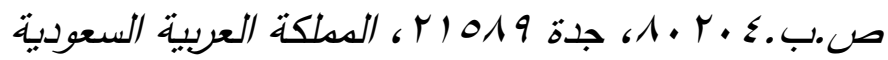 \\ arushdi@kau.edu.sa
}

العستخلص. تشتمل الطرائق اليدوية لحل المعادلات البولانية الكبيرة

على طرائق جبرية بحتة وأخرى تستخدم خريطة كارنوه متغيرة المحتويات

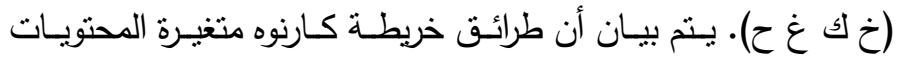
تتافس الطرائق الجبرية البحتة (وتتفوق عليها أحيانا) لأنها: (أ) تشتنل

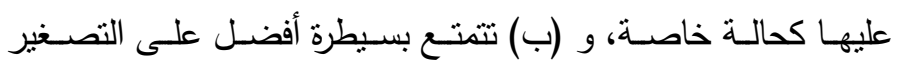
الأعظمي لتمثيل الدوال المعنية، ومن ثم فإنها أقدر على إنتاج حلول عامة أصغر وأبسط.

الكلمات الدالة : حل المعادلات البولانية الكبيرة، طرائق الخريطة متغيرة المحتويات، الطرائق الجبرية البحتة. 